\title{
Polymer Degradation: Category, Mechanism and Development Prospect
}

\author{
Yuanxin $\mathrm{He}^{1, \mathrm{a},{ }^{*}, \dagger}$, Hongyu $\mathrm{Li}^{1, \mathrm{~b},{ }^{*}, \dagger}$, Xiang Xiao ${ }^{1, \mathrm{c},{ }^{*}, \dagger}$ and Xinyu Zhao ${ }^{1, \mathrm{~d}, *, \dagger}$ \\ ${ }^{1}$ School of Queen Mary University of London Engineering School, Northwestern Polytechnical University, 710100 Xi'an City Shaan Xi \\ Province, China \\ ${ }^{\dagger}$ These authors contributed equally to this work.
}

\begin{abstract}
With the increasing demand for polymers, white pollution has become a serious concern all around the world. The admirable degradation methods of them are desirable for overcoming this problem. In the past several decades, numerous researches on polymer degradation have been reported. This review commits to different degradation strategies of polymers and four main degradation protocols firstly, including photodegradation, oxidative degradation, catalytic degradation, and biodegradation, are demonstrated in detail. Secondly, some specific samples are discussed for each kind of degradation. Finally, the outlook and future of polymer degradation are proposed. In particular, the comprehensive comparison of different degradation methods is covered to provide the best choice for dealing with different polymers wastes. These will be beneficial to the development of processing plastic and conversion of polymer wastes.
\end{abstract}

\section{Introduction}

With the rapid development of science and technology, polymer materials have been widely used in people's daily life and industrial production $[1,2]$. The birth of functional polymer materials also promotes the development of industrial production, agricultural production, medical and health fields in the direction of depth and refinement. According to IHS data, the global demand for polycarbonate in 2019 is about 4.4 million tons, and the total production capacity is about 5.6 million tons. Annual capacity is expected to increase by 42 percent over the next several years. It will increase from 5.6 million tons in 2019 to 8 million tons in 2024. Although polymer materials bring great convenience to human life, the hardly degradable polymer materials represented by "white pollution" also cause great biological harm and environmental pollution [3]. Therefore, how to degrade the polymer materials after use has become an important research topic for academic and industrial fields.

At present, polymer materials are mainly disposed of utilizing incineration, heap burial, and degradation. However, due to the toxic gases and greenhouse effect of burning, this method has been strictly regulated [4]. At the same time, with the serious white pollution and biological environment problems caused by heap burial, it is not an effective way of long-term disposal of waste polymer materials [5]. Therefore, the development of degradable polymer materials and the promotion of the development of polymer degradation technology have become important methods to dispose of waste polymer materials.

Many types of research have been conducted in the field of polymer degradation. Recent theoretical developments have shown that there are mainly four methods for polymer degradation, including thermal degradation, photodegradation, catalytic degradation, and biodegradation. Generally, energy from heat and light could be absorbed by the chains in polymer, which cause a series of chain-breaking reactions, leading to thermal cracking and light cracking, respectively [6,7]. The presence of oxygen would promote these two reactions because several types of radicals would be formed by oxidation, which can react with other chains [6,7]. Except for oxidation, another effective method for promoting cracking reaction is to use a catalyst, such as zeolite, which could further improve the reaction activity and produce value-added chemicals [8]. Furthermore, biodegradation is a very promising strategy, which is much more complex. Microorganisms such as bacterial could produce enzymes to convert polymer into low molecular weight fragments by hydrolysis and oxidative reactions, and then "eat" them and excrete small molecules like carbon oxide and water [9].

Understanding the mechanism of different cracking strategies is supposed to help people find better methods to dispose or recycle waste polymeric materials. Based on giving an overarching framework of polymer degradation approaches for beginners or providing systematic collection to support further study in this field, this work will review the mechanism and features of basic polymer cracking ways and make a comparison between them. The paper could provide important theoretical knowledge for readers to learn about or deepen their understanding of the degradation of the polymer.

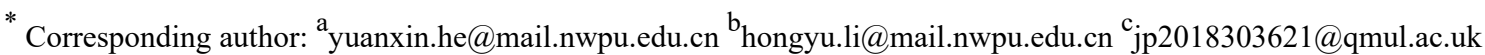

$\mathrm{d}_{\text {zhaoxinyu@mail.nwpu.edu.cn }}$
} 


\section{Degradation strategy}

\subsection{Thermal degradation}

Heat is one of the most common types of energy in nature. Thermal energy could promote the movement of the polymer chain, leading to the degradation and decompose of the polymer, as well as the decrease in a series of properties, such as mechanical property. At certain elevated temperatures, polymers' the physical and chemical properties of polymers will be changed spontaneously $[6,10]$. The chemical bonds absorb enough energy from heat in polymers, resulting in the breaking of these chemical bonds and then producing some free radicals. The formed free radicals are extremely active, and they would react with the molecules around. Hence, more chains can be further broken, and more free radicals are generated. Subsequently, many parameters of polymers would be changed, including molecular weight, molar weight distribution, degree of crystallinity, degree of branching, and so on. As the reaction process, the variation in mass, state, shape even color can be observed [6]. As a result, the working performance, such as mechanical or thermal performance of the polymers, would alter dramatically, being prone to cause the failure of the material [6].

$\mathrm{Xu}$ et al. have carefully investigated the thermal degradation mechanism of PVC material, indicating the products, include different hydrocarbons, are produced with $\mathrm{HCl}$ in the gas phase [11]. The team of Cleide Borsoi took Flynn-Wall-Ozawa method to explore the thermal degradation of cellulose nanofibers. The results showed that the degradation behaviors of cellulose are affected not only by morphology, crystallinity and degree of polymerization but also mechanical defibrillation and drying by lyophilization [12]. Wang et al. have verified that bonging temperature, dwell time, and thermal conductivity of mold material influence the cracking rate of nonwovens(PP/PET fiber) using FTIR and DSC methods [13].

\subsubsection{Thermal degradation without oxygen}

Even though it is almost impossible to ignore the presence and function of oxygen in terms of the chain-breaking reaction, the study in this field is still vital since plenty of heat treatments on polymeric materials are operated under non-oxygen condition, and the degree of thermal degradation determines the quality of the products directly $[6,14,15]$. There are mainly three types of methods for thermal degradation [16].

\section{(1) Depolymerization}

Depolymerization is also called unzippering reaction. The cracking starts at the end or the weak points of the main chains, and then the connecting monomers, which are the only products theoretically, break away from the backbones one by one [16]. For instance, the thermal cracking of polymethyl methacrylate (PMMA), as shown in Fig. 1a.

\section{(2) Random chain-breaking}

Random chain-breaking reaction or random scission reaction could start from any points in polymer chains. The main product is an oligomer, which refers to low molecular weight polymers [16]. For example, the thermal decomposes of polyethylene (PE).

In reality, for most polymers, the 2 methods of cracking above always happen simultaneously (Fig. 1b).

\section{(3) Side group elimination}

Side group elimination is sometimes called small molecule elimination. The reactions always begin as the elimination of side groups, producing small molecules instead of monomers. The formed small molecules are based on the groups attached to the polymer chains [16]. For example, hydrogen chloride $(\mathrm{HCl})$ is formed at the early stage in the thermal cracking of polyvinyl chloride (Fig. 1c).

With the process of elimination, more and more weak points on the backbone would be exposed, and then the depolymerization and random chain-breaking reactions of polymers spread from these exposed weak points [16]. (a)

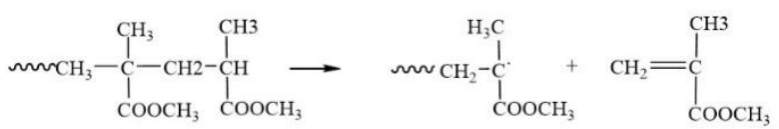

(b)

$\sim \mathrm{CH}_{2}-\mathrm{CH}_{2}-\mathrm{CH}_{2}-\mathrm{CH}_{2}-\mathrm{CH}_{2} \sim n \rightarrow \sim \sim \mathrm{CH}_{2}-\mathrm{CH}=\mathrm{CH}_{2}+\mathrm{CH}_{2}=\mathrm{CH} \sim m$

(c)

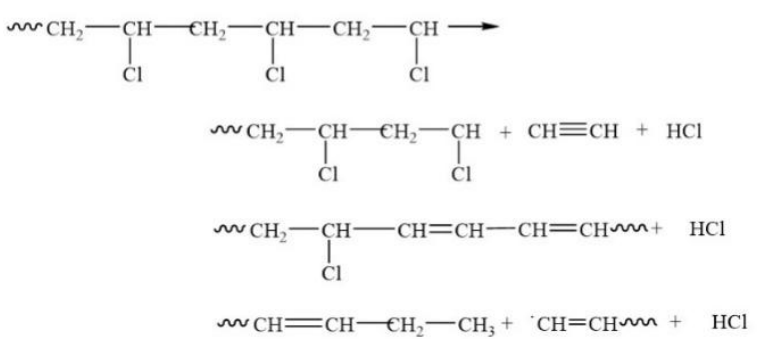

Fig. 1. Thermal cracking mechanism of (a) PMMA, (b) PE, and (c) PVC.

\subsubsection{Thermal-oxidative degradation}

In the processing, storage, and application of polymeric materials, contact with oxygen can hardly be avoided. In normal thermal degradation, some radicals formed from cracking could react and recover to chains again if the temperature is not high enough [8]. However, oxygen could react with these radicals to prevent them from forming polymer chains again, so the reaction rate can be increased with the function of oxygen. It is proved that high-density polyethylene (HDPE) cracking is faster at a considerably lower temperature in the air than that in a nitrogen atmosphere [16].

The mechanism of thermal-oxidative degradation is a sort of typical chain-step radical process, with initiation, propagation, and termination. The main feature is the"auto-oxidative" reaction [14]. 
(1) Mechanism-initiation (Fig. 2a)

With the function of heat and oxygen, the basic radical $\mathrm{R} \cdot$ forms.

(2) Mechanism-propagation (Fig. 2b)

$\mathrm{R}$ - can react with oxygen then other chains, forming a peroxide, $\mathrm{ROOH}$, which could decompose and produce new radicals to react with more other chains to obtain more basic radicals.

(3) Mechanism-termination (Fig. 2c)

Different radicals may react with each other, leading to coupling termination.

(a)

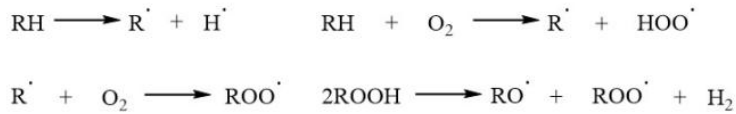

(b)

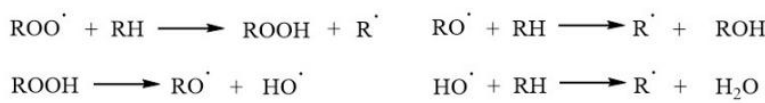

(c)

$$
\begin{aligned}
\mathrm{ROO}^{\circ}+\mathrm{ROO}^{\circ} & \longrightarrow \mathrm{ROOR}+\mathrm{O}_{2} \\
\mathrm{ROO}^{\circ}+\mathrm{R}^{\circ} & \longrightarrow \mathrm{ROOR} \\
\mathrm{R}^{\circ}+\mathrm{R}^{\circ} & \longrightarrow \mathrm{R}-\mathrm{R}
\end{aligned}
$$

Fig. 2. Reaction mechanism of thermal-oxidative degradation [14].

\subsection{Photodegradation}

Photodegradation refers to the phenomenon of chain fracture caused by the existence of photosensitive groups in the polymer chains such as carboxyl, carbonyl, and unsaturated bonds due to the action of light and the presence of oxygen [7]. It is an important kind of method for polymer degradation in our daily life. Light is usually used as the source to provide energy for electrons in the polymer, and the polymer will be excited by light to promote the following chain-breaking reaction. Subsequently, the molecular weight of the polymer will decrease significantly. In the reaction process of polymer chain-breaking step by step, various properties of the polymer, such as mechanical properties and chemical properties, will also gradually change, which could achieve the degradation of different polymers to protect the environment.

\subsubsection{Photodegradation without oxygen}

Photodegradation without oxygen is the basic reaction of photodegradation. The photodegradation of polymers could be divided into two classes, including direct degradation and indirect degradation. For the direct degradation, the polymer could absorb photons directly, and polymer molecules will be directly transformed from the ground state to the excited state [16]. During this process, polymer molecular chains could be broken, and molecular weight gradually decreases. While for an indirect degradation process, the energy of photons is absorbed by a substance in the environment, firstly, which could promote the substance to an excited state. And then, the polymer molecules are induced to an excited state, which will result in the degradation of polymers by breaking down the chains.

There are two main types of chemical reactions during the degradation.

\section{(1) Depolymerization}

The photons with enough energy (especially for UV light) can break the chemical bonds, which can induce the generation of free radicals [17]. As shown in Fig.3a, polymer chains with free radicals are active. Some of them would release monomers from the end with free radicals under the attack of photons.

\section{(2) Random chain-breaking}

There are no free radicals for some polymers in the end, or chemical bonds in the polymer chains have similar energy. In this case, the photon with enough energy will randomly break the bonds of them, introducing free radicals and undergoing further reaction (Fig. 3b).

(a)

$$
\begin{aligned}
\sim M-M-M-M-M^{*} & \longrightarrow \sim M-M-M-M^{*}+M \\
\sim & \sim M-M-M-M^{*} \longrightarrow M M-M-M^{*}+M \\
\sim & \longrightarrow M-M-M^{*} \longrightarrow \sim M-M^{*}+M
\end{aligned}
$$

(b)

$$
\begin{aligned}
& \sim \Omega M-M-M-M-M-M \sim \Omega \\
& \downarrow \mathrm{h} v \\
& \sim \sim M-M-M \cdot+\cdot M-M-M-M-M \sim \sim
\end{aligned}
$$

Fig. 3. The reaction mechanism of Depolymerization and Random chain-breaking $[6,17]$.

\subsubsection{Photo-oxidative degradation}

The effect from oxygen is always hard to avoid [18]. Therefore, it is vital to focus on photo-oxidative degradation. Generally, photo-oxidative degradation goes through three steps as following (Fig. 4).

(1) Mechanism-initiation. Due to the function of light and oxygen, the basic radical $\mathrm{R}$. is generated, which will be used in the next propagation process [10].

(2) Mechanism-propagation. $\mathrm{R}$. react with oxygen and form a peroxide $\mathrm{ROOH}$, which could decompose and produce new radicals. These new radicals decomposed by $\mathrm{ROOH}$ can go on next reactions. After that, there will be more new radicals produced and reacted with each other [10].

(3) Mechanism-termination. Different radicals may react with each other, leading to coupling termination [14]. 


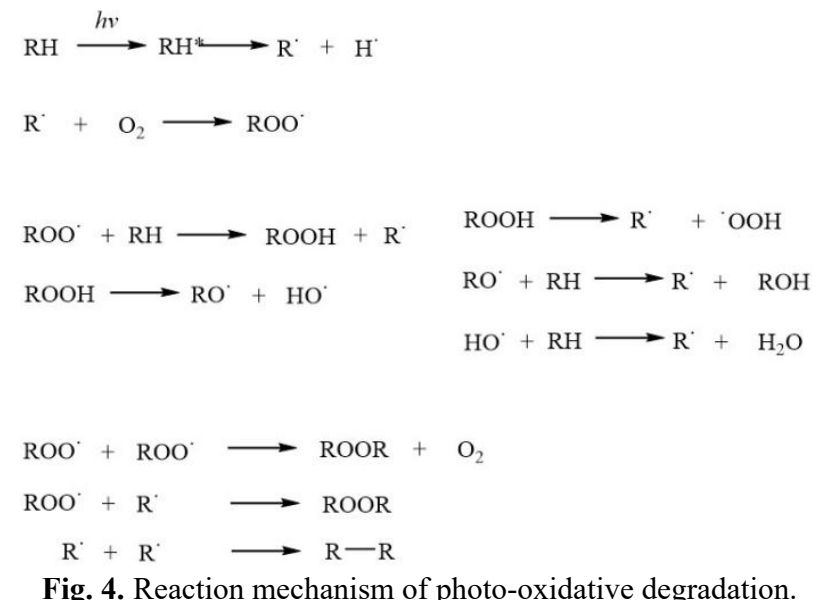

\subsubsection{Source of photo-oxidative degradation}

Furthermore, some special structures in the polymer may affect reactions.

(1) $\mathrm{ROOH}$ - the very beginning of photo-oxidative degradation.

$\mathrm{ROOH}$ can be generated from photo-oxidative degradation. The radical $\mathrm{ROOH}$ can accumulate and decompose [10]. After the decomposition of $\mathrm{ROOH}$, many radicals can be obtained (Fig. 5).

(2) Metal ions - sensitizer for the photo-oxidative degradation of polyolefin.

Metal ions, again, could work as catalysts, which are used to increase or decrease the rate of reactions. The photo-oxidative degradation of PP is related to the photosensitizer effect of the residual catalyst, which can take part in the reaction in the form of radical ions generated via redox of the excited state $[10,19]$.

(3) Carbonyl groups - Norrish reaction

Moreover, if there are carbonyl groups in the polymer, a series of unique reaction called Norrish reaction may occur, including an $\alpha$ chain-breaking, $\alpha-\beta$ chain-breaking, and $\beta$ hydrogen transfer reaction [8]. As shown in Fig. 6, these reactions could provide more position for the initiation for photo-degradation reaction.

Regeneration of $\mathbf{R}^{\text {. }}$
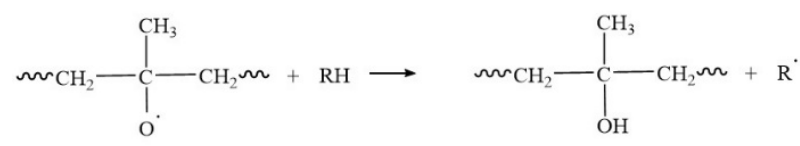

Formation of $\mathbf{R}^{\text {. }}$

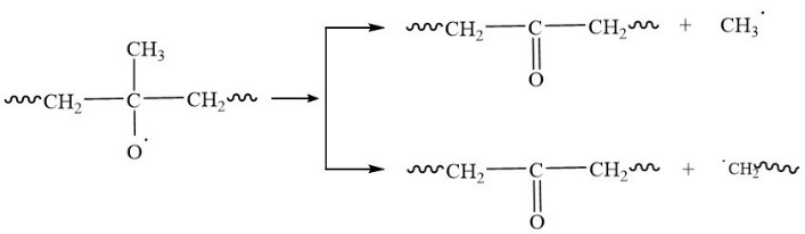

Fig. 5. The production and decomposition of ROOH in photodegradation reactions [10].

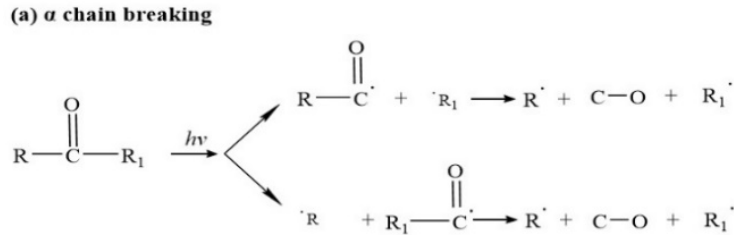

(b) $\alpha-\beta$ chain breaking

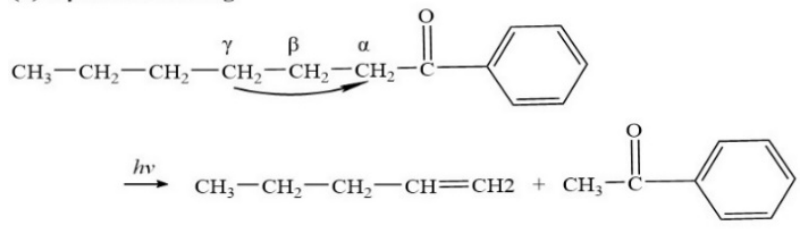

(c) $\beta$ hydrogen transfer reaction

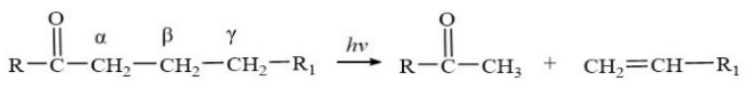

Fig. 6. Three different Norrish reactions for photo-degradation reaction [8].

\subsection{Catalytic degradation}

\subsubsection{Oxidative degradation}

With the development of population and technology, the demand for light alkenes such as propene and ethene grows with each passing day. The main method of producing light alkenes is steam cracking of plastic polymers, which wastes a lot of energy and emits much $\mathrm{CO}_{2}$. Compared with steam cracking, oxidative catalytic cracking shows a promising future, which not only decreases the heat temperature but also produces some value-added chemicals.

The existence of oxygen will change the reaction thermodynamics, inhibit heavy productions, and accelerate reaction during oxidative alkene cracking. Besides, cracking is initiated by the single bond between two carbon or the formation of radicals. Oxygen presents in the initiation reaction during the oxidative cracking of the gas phase. It could oxidize part of the alkene and generate water and $\mathrm{CO}$. Besides, it can also produce heat for the next stage [8].

Using catalyst during oxidative cracking needs lower temperature relative to not using a catalyst, which also adjusts the selectivity of forming light alkenes. Thus, a catalyst must promote the production rate. For example, with catalyst $\mathrm{Li} / \mathrm{MgO}$, the cracking rate of hexane could increase from $1 \mathrm{wt} \%$ to $17 \mathrm{wt} \%$ at the same conditions [8].

Besides, the mechanism of oxidative catalytic cracking could be divided into two steps. And the initiation mechanism largely depends on the active positions of the catalyst. The formation of alkyl radicals and extraction of hydrogen from alkane initiate the surface. This will be a $\beta$-scission reaction, and cracking productions will be produced. For example, the catalysts could be gold-containing, oxychlorides, and alkali. Under the same conditions, the gold-supported catalyst has higher propane conversion and selectivity to olefins than 
the platinum-supported catalyst [20]. The $\mathrm{Ce}_{0.5} \mathrm{Zr}_{0.5} \mathrm{O}_{2}$ has shown a slight activity. However, when the catalyst was deposited with $0.2 \mathrm{wt} \%$ gold, an increase in catalytic activity was observed. A further improvement in catalytic activity was achieved with gold-supported sulphated $\mathrm{Ce}_{0.5} \mathrm{Zr}_{0.5} \mathrm{O}_{2}$ [20].

\subsubsection{Zeolite degradation}

Zeolite is a common acid solid catalysis, which is widely used for polymer cracking to get olefin. Light alkene has developed into the footstone of the petrochemical industry. Thus, there is a huge requirement for ethylene and propylene. The main method for producing them is the cracking of petroleum. The depletion of fossil resources has put tremendous stress on the production that relies on oil cracking. However, less than $9 \%$ of plastics have been recycled [21]. Under the circumstances, improving the recovery is necessary. Converting plastics selectively to light lubricants and fuels provides a new but reliable solution to plastics recycling. By zeolite cracking, olefins could be produced from polymer materials.

The architecture and morphology of zeolite influence the catalysis process. Lower temperature and less energy are needed with zeolite cracking. By employing zeolite catalyst, the reaction activity could be further improved. The activation energy of polymer cracking could be decreased, which has been carefully studied by Zhao and co-authors to crack LDPE with different morphology MFI zeolites [22]. Besides, the side branches MTW zeolites have also been used to crack LDPE, which could mainly produce the gasoline, compared with the conventional MTW zeolite [23,24]. Because of the selectivity of the microporous network, which makes it is possible for the selectivity of the product. For example, HY gives monomers a selectivity range, whose product has less diesel and aliphatic hydrocarbons, and naphthene [24].

\subsection{Biodegradation}

Biodegradable polymer refers to a kind of polymer, which can be completely degraded with the aid of microorganisms like bacteria or can be dissolved into water and degraded by $\mathrm{CH}_{4}$ (methane), $\mathrm{CO}_{2}$, and metal-salt. Until now, the most common materials used are polylactic acid (PLA), polyvinyl alcohol (PVA) and polymethyl succinate (PBS) [25]. With the development of biodegradation technology, environmental problems can be gradually solved largely. At the same time, these wastes can be converted into different useful materials or chemicals, which could further promote the development of economics and life.

In recent years, the biodegradable polymer has been widely used as environmental-friendly protection materials (Mulch for agriculture, construction), packaging materials (Packaging film, garbage bags, food packaging bags), biologically functional materials, medical materials (bandages, surgical sutures), and many other fields. It also shows unique advantages compared with single-use plastic products.

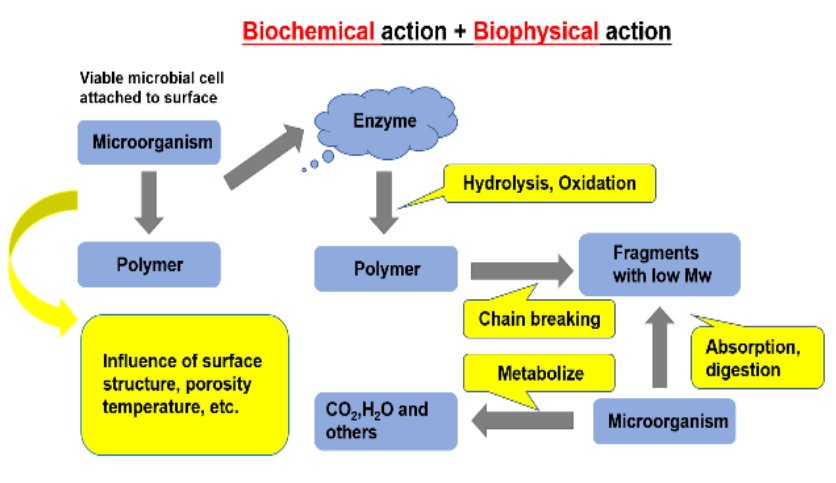

Fig. 7. Schematic diagram of biodegradation [9].

\subsubsection{Mechanism of biodegradation}

Biodegradation is a very complex process. It is a combination of biochemical and biophysical action, and still now, most of the mechanisms are not very well studied.

The process of biodegradation can be mainly divided into three stages (Fig.7). First, bacteria will attach to the surface of the polymer products. The surface structure and temperature will influence this process. For example, both superhydrophobic and super hydrophilic polymer surfaces increase bacterial absorbability. Secondly, the polymer chain will be broken by the enzyme secreted by bacteria with hydrolysis and oxidation reaction $[9,26]$. Then, Fragments with low MW (<500) will be absorbed and digested by bacteria. It goes through a series of metabolizing processes and eventually gets rid of it in carbon dioxide, water, and other substances [9].

\subsubsection{Classification of biodegradable polymers}

There are two ways to identify the types of biodegradable polymers. One is based on the degradation mechanisms and damage form of the polymer. The other one is based on the mode of production [27].

(1)According to the degradation mechanisms and damage form, biodegradable polymers are divided into completely biodegradable polymers and bio-destructive polymers, which could only be degrading to random fragments by microbial.

(2)According to the mode of production, there are three types of biodegradable polymers. It includes macromolecule production by microorganisms (PHBV, PHB), synthetic polymer material (PLA, PCL), and natural polymer materials (cellulose, starch, lignin, chitin). One of the more promising polymers is additive biodegradable plastics. This kind of plastic is made by adding biodegradable ingredients as additives to raw materials (PE, PP, PS) at a relatively low price [27]. 


\subsubsection{Some specific biodegradable polymer materials}

\section{Polylactic acid}

Polylactic acid (PLA) is one of the most biodegradable thermoplastic materials. It is made from starch, honey, and other raw materials through fermentation. The polymer was obtained by further chemical synthesis [28].

PLA degrades more easily than other plastics. Under some environmental conditions, PLA can hydrolyse after contacting water. The whole process does not require sunlight, and it is not sensitive to $\mathrm{pH}[28,29]$. This is the big advantage of PLA as a biodegradable polymer material. At present, there are numbers of relevant PLA research being carried out, and some technologies have already entered the promotion stage. Some of the applications include extrusion sheets, injection moulding, blister products, spinning, bidirectional stretch film, and other processes. PLA is also widely used in medical treatment because of its good antimicrobial properties.

\section{Polyhydroxy fatty acid esters}

Polyhydroxy fatty acid esters (PHA) is a kind of biological polyester particles synthesized by microorganism. PHA has good biocompatibility, but its application has been greatly limited due to its poor mechanical properties and high cost [30].

PHB is another kind of PHA, which is widely used. It has a simple structure and can be completely decomposed within 12 months, only releasing pollution-free water and carbon dioxide. Therefore, PHB has become one of the preferred materials for green packaging.

\section{Conclusion}

In this paper, various problems and countermeasures encountered in the development of polymer degradation in recent years are summarized. Then, introducing the most important degradation types in the four fields of polymer degradation in detail makes a certain interpretation for the basic mechanisms and prospects of polymer degradation. In summary, the paper shows thermal degradation, photodegradation, catalytic degradation, and biodegradation. Thermal degradation (including thermal-oxidative degradation) is a common type of degradation. This paper expounds on the main mechanism and prospect of thermal degradation. It is difficult to control temperature and other conditions, such as pressure, required for degradation in real life. In the process of polymer thermal degradation, other substances such as additives can also participate in the response, causing the discharge of environmental pollutants. This is the problem thermal degradation is going to be solved. Photodegradation is relatively inexpensive, and the light sources needed for degradation are readily available. It is more environmental-friendly and has a very broad market. The research direction of photodegradation provides corresponding higher efficiency photodegradation reactions for different polymers. For example, different photosensitizer catalysts can be added to different polymers and improve the polymer structure to make it more photodegradable. Compared with steam cracking, catalytic oxidation degradation reduces the temperature required for the reaction, produces chemicals with added value, and meets the increasing demand for light olefin such as propylene and ethylene, which has a good application prospect. Biodegradation has shown its incomparable advantages in many fields, and it is a promising direction for the future development of polymer materials. But at the present stage, there still are some problems that need to be solved, such as high material cost, limited material uses, and lack of production technology. New degradation technology will reduce environmental pollution and make a breakthrough in navigation, aerospace, agriculture, medical instruments, biological applications, clean energy, and many other fields.

These summaries and explorations can provide a very good reference for people to understand the mechanism and development prospects of polymer degradation. At the same time, it can also provide a specific reference for the degradation of different types of polymer.

\section{References}

1. Shahinpoor, M.; Kim, K. J. Smart Mater. Struct. 2005, 14 (1), 197.

2. Alrifaiy, A.; Lindahl, O. A.; Ramser, K. Polymers 2012, 4 (3), 1349- 1398.

3. Nicolas, J.; Mura, S.; Brambilla, D.; Mackiewicz, N.; Couvreur, P. Chem. Soc. Rev. 2013, 42 (3), $1147-$ 1235.

4. E. Chiellini, R. Solaro Adv Mater, 1996, 8, 305-313

5. Y.M. Zhang, J.M. Jiang, Y.M. Chen Polymer, 1999, 40 (22), 6189-6198

6. Ray, Sudip. Handbook of Environmental Degradation of Materials || Thermal Degradation of Polymer and Polymer Composites. 2018, 185-206.

7. Kai Zhang, Amir Hossein Hamidian, Aleksandra Tubić, YuZhang, James K.H. Fang, Chenxi Wu, Paul K.S. Lame. Envriomental Pollution, 2021,274, 116554.

8. Boyadjian, C. , \& Lefferts, L. . (2018). European Journal of Inorganic Chemistry.

9. A Moses, A. E. , EB Bassey, \& Udoh, S. B. Risk Factors Associated With Oral Cavity Colonization By Candida Albicans, Among Immunosuppressed Persons In Uyo, South South Nigeria. Journal of Medical Laboratory Science, 2012, 21

10. Cuadri, A. A. , \& JE Martín-Alfonso. Polymer Degradation and Stability, 2017, 141, 11-18.

11. Zhuo, X. A. , Ssk, A., Sz, A., Bz, A. , \& MD B. Polymer Degradation and Stability, 176.

12. Borsoi, C. , Zimmernnam, M. , Zattera, A. J. , Santana, R. , \& Ferreira, C. A. . (2016).. Journal of Thermal Analysis and Calorimetry.

13. Wang, X. Y. , \& Gong, R. H. Journal of Applied Polymer Science, 2007, 104(1), 391-397. 
14. Rabek, Jan F. [Comprehensive Chemical Kinetics] Degradation of Polymers Volume 14 || Chapter 4 Oxidative Degradation of Polymers. 1975, 425-538.

15. Mcneill, I. C. Polymer Engineering and Science, 1980, 20(10), 668-674.

16. Pielichowski, K. , \& Njuguna, J. Thermal Degradation Of Polymeric Materials. 2008.

17. S.M.A. Abel, K. Werner, Z. Christiane, H. Stefan, C. R M .Global Change Biol., 2018, 24 (4), 1405-1416.

18. M Galloway, T. S. , \& Lewis, C. N. . (2016). Proceedings of the National Academy of Sciences of the United States of America, 2331.

19. R. Stephen Davidson, Dean Goodwin, Ph. Fornier de Violet. Tetrahedron Letters 1981, 22(26), 2485-2486

20. Narasimharao, K. \& Ali, T. T.. Catalysis Letters, 2013, 143(10), 1074-1084.

21. Liu, S., Kots, P. A., Vance, B. C., Danielson, A., \& Vlachos, D. G. .Science Advances, 2021, 7(17), eabf8283.

22. Blay, V., Louis, B., Miravalles, R., Yokoi, T. , Peccatiello, K. A. , \& Clough, M. ACS Catal. 2017, 7, 6542-6566..

23. Zhao, Y., Ye, Z., Zhang, H., Zhang, Y., \& Tang, $Y$. Industrial \& Engineering Chemistry Research, 2019, 58(29), 13174-13181.

24. Zhao, Y., Ye, Z., Wang, L., Zhang, H., F Xue, \& Xie, S. Crystal Growth \& Design, 2018, 18, 2, 1101-1108.

25. Bassey, E., Mo, B. C., Aluyi, H. , \& Anukam, K. . Journal of Medical Laboratory Science, 2004,13(1).

26. Mathew C. Celina, Jeffrey S. Wiggins, Norman C. Billingham., Polymer Degradation and Performance, ISBN: 978-0-8412-6978-1, Oxford University Press, 2009,67-75.

27. Taghavi Navid, Udugama Isuru Abeykoon, Zhuang Wei-Qin et al. Biotechnology Advances, 2021, 21-35.

28. Bordes, P., Pollet, E. , \& Averous, L.. PROGRESS IN POLYMER SCIENCE. 2009.

29. HAMAD K,KASEEM M,AYYOOB M, Progress in Polymer Science,2018,85, 85-127.

30. Chuah JA, Tomizawa S. Yamada M, Appl Environ Microbiol,2013,79(12), 813-3821. 